\title{
Genome-Wide Characterization and Gene Expression Analyses of GATA Transcription Factors in Moso Bamboo (Phyllostachys edulis)
}

\author{
Taotao Wang ${ }^{1}$, Yong Yang ${ }^{1}$, Shuaitong Lou ${ }^{2}$, Wei Wei ${ }^{2}$, Zhixin Zhao ${ }^{3}$, Yujun Ren ${ }^{2}$ (D), \\ Chentao Lin $^{4}$ and Liuyin Ma 1,2,*(D)
}

1 Basic Forestry and Proteomics Research Center, College of Forestry, Fujian Provincial Key Laboratory of Haixia Applied Plant Systems Biology, Fujian Agriculture and Forestry University, Fuzhou 350002, China; fjnlwtt@163.com (T.W.); yongwithyang@163.com (Y.Y.)

2 Fujian Provincial Key Laboratory of Plant Functional Biology, College of Life Sciences, Fujian Agriculture and Forestry University, Fuzhou 350002, China; Loushuaitong@163.com (S.L.); wwei_0501@163.com (W.W.); ryj@fafu.edu.cn (Y.R.)

3 College of Biopharmaceutical and Food Engineering, Shangluo University, Shangluo 726000, China; zxzhao@slxy.edu.cn

4 Department of Molecular, Cell and Developmental Biology, University of California, Los Angeles, CA 90095, USA; clin@mcdb.ucla.edu

* Correspondence: 1ma223@fafu.edu.cn

Received: 13 November 2019; Accepted: 16 December 2019; Published: 18 December 2019

\begin{abstract}
Moso bamboo is well-known for its rapid-growth shoots and widespread rhizomes. However, the regulatory genes of these two processes are largely unexplored. GATA transcription factors regulate many developmental processes, but their roles in moso bamboo height control and rhizome development remains unexplored. Here, thirty-one bamboo GATA factors (PeGATAs) were identified, which are evolutionarily closer to rice than Arabidopsis, and their gene expression patterns were analyzed in bamboo development and phytohormone response with bioinformatics and molecular methods. Interestingly, PeGATAs could only be classified into three groups. Phytohormone responsive cis-elements were found in PeGATA promoters and the expression profiles showed that PeGATA genes might respond to gibberellin acid and abscisic acid but not to auxin at the transcriptional level. Furthermore, $P e G A T A$ genes have a tissue-specific expression pattern in bamboo rhizomes. Interestingly, most $P e G A T A$ genes were down-regulated during the rapid-growth of bamboo shoots. In addition, over-expressing one of the PeGATA genes, PeGATA26, significantly repressed the primary root length and plant height of transgenic Arabidopsis plants, which may be achieved by promoting the gibberellin acid turnover. Overall, our results provide insight into the function of GATA transcription factors in bamboo, and into genetic resources for engineering plant height.
\end{abstract}

Keywords: GATA genes; transcription factor; gene expression; rhizome development; shoot rapid-growth; moso bamboo

\section{Introduction}

Moso bamboo is one of the most abundant non-timber forestry species and provides important resources for food, architecture, papermaking, and fiber [1]. More importantly, moso bamboo is known for its explosive shoot growth rate, with a peak growth rate of $1 \mathrm{~m}$ per day [1]. The rapid-growth shoot is largely dependent on the widespread rhizome system, which provides nutrients by absorbing from the soil, and more importantly, transporting from other rhizome-connected adult bamboos [2]. Therefore, studying the development of shoots and rhizomes will help us to understand the rapid-growth 
regulation of bamboo, and provides effective candidate genes for genetic manipulation of plant height in crop and forestry species.

The GATA factors play important roles in many developmental processes by binding to the consensus DNA sequence (A/T)GATA(A/G) to regulate gene transcription [3,4]. The GATA factors have a highly conserved type IV zinc finger DNA binding domain $\left(\mathrm{CX}_{2} \mathrm{CX}_{17-20} \mathrm{CX}_{2} \mathrm{C}\right)$, followed by a basic region [5-7]. In animals, GATA factors typically contain two zinc finger domains $\left(\mathrm{CX}_{2} \mathrm{CX}_{17-20} \mathrm{CX}{ }_{2} \mathrm{C}\right)$, while only the C-terminal domain has a DNA binding function [5]. Animal GATAs are involved in development, differentiation, and control of cell proliferation [7]. However, the fungal GATA factors only contain a single zinc finger domain that is highly similar to the $\mathrm{C}$-terminal zinc finger domain of the animal GATA factors [4,8]. In plants, GATA factors contain a $\mathrm{CX}_{2} \mathrm{CX}_{18} \mathrm{CX}_{2} \mathrm{C}$ or $\mathrm{CX}_{2} \mathrm{CX}_{20} \mathrm{CX}{ }_{2} \mathrm{C}$ zinc finger domain $[9,10]$. Interestingly, most plant GATA factors have a single zinc finger domain, but very few of them contain two zinc finger domains [9-11].

In animals, GATA factors are involved in cell differentiation and organ development. Mutations in animal GATA factors cause severe developmental disorder diseases including anemia, deafness, and renal and cardiac defects [12]. Fungal GATA factors play roles in nitrogen control, siderophore biosynthesis, light-regulated photomorphogenesis, and circadian regulation [4].

Plant GATA factors originate from the identification of GATA motifs in regulatory regions of light and circadian clock responsive genes [13]. The first plant GATA factor was identified from Nicotiana tabacum [14]. GATA factors have been identified in many plant species, including Arabidopsis (29), rice (28), apple (35), and soybean $(64)[9,10,15]$. Plant GATA factors are involved in many developmental processes, affecting plant architecture [16], flower development [17], hypocotyl elongation [18], and seed germination [19]. Plant GATA factors mainly regulate nitrogen metabolism [14,20] and plant growth [21-23]. They regulate plant growth in two ways: either directly downstream of phytohormone signal transduction [23] or acting as a transcriptional regulator by integrating both light and phytohormone signals [21,22].

Plant GATA factors regulate light signal transduction by combining with the GATA motif in the promoter of light-related genes [24,25]. GATA2 (At2g45050) has also been identified as a key transcriptional regulator of the integration of light and brassinosteroid signaling pathways [22]. Recent evidence suggests that GATA factors are involved in the regulation of plant hormone signal transduction. Two orthologous GATA-type transcription factors-GNC (Gata, nitrate-inducible, carbon metabolism-involved) and GNL (GNC-like) from Arabidopsis-were identified as GA-regulated genes [21,23]. Loss-of-function mutants and over-expression lines of GNC and GNL are functionally related to germination, greening, and flowering time [17]. CHIP (chromatin immunoprecipitation) results show that these two genes are direct targets of PIF transcription factors. gnc and gnl loss-of-function mutations suppress ga1 phenotypes, supporting GNC and GNL as important repressors of GA signaling [21]. Another important phytohormone, auxin, is also regulated by GNC and GNL through functioning downstream of ARF2 (Auxin Response Factor 2) [23]. In addition, the GATA factors are induced by cytokinin [26]. These results indicate that GATA factors play crucial roles in plant development and phytohormone-mediated growth. However, the role of GATA factors in rapid-growth and rhizome development remains elusive.

Recently, large-scale transcriptome analysis has shown that light and phytohormones may play important roles in the rapid-growth of bamboo [27-29]. In addition, a large number of transcription factor families, involved in the abiotic stress response and flower development, have been studied in moso bamboo [30-32]. Although a previous study has functionally characterized the rapid-growth associated gene-PeGSK1 [1], the rapid-growth associated transcription factor families are largely unexplored in moso bamboo.

In this study, we performed a genome-wide survey of GATA factors in moso bamboo. A total of 31 GATA factors were identified in the moso bamboo genome. The phylogenetic relationship, gene structure and conserved domains of moso bamboo were systematically analyzed. The phytohormone-related cis-elements and gene expression of PeGATAs under GA (gibberellin 
acid), ABA (abscisic acid), and auxin treatment were also characterized. More importantly, the gene expression of PeGATAs in different rhizome tissues and rapid-growth shoots were analyzed in detail. In addition, one growth related PeGATA-PeGATA26-was over-expressed in Arabidopsis to functionally validate its role in regulating plant height. Overall, our results provide information on the involvement of GATA factors in rhizome tissue development and rapid-growth shoots.

\section{Results}

\subsection{Genome-Wide Characterization of GATA Factors in Moso Bamboo}

A total of 31 potential GATA factors were identified in moso bamboo and named PeGATA1 to PeGATA31 based on the chromosomal location. The CDS (Coding regions) and protein sequences of PeGATA genes are listed in Tables S1 and S2. The detailed information of these PeGATA factors including length of CDS, size of amino acid, molecular weight (MW) of protein, gene location on chromosome, and isoelectric point (PI) are listed in Table 1.

Table 1. GATA factors in moso bamboo.

\begin{tabular}{|c|c|c|c|c|c|c|c|}
\hline Name & Gene ID & Location & ORF Length (bp) & Size (aa) & MW (kDa) & PI & Group \\
\hline PeGATA1 & PH01000001G0820 & $\begin{array}{l}\text { 603239-605821 } \\
\text { (- strand) }\end{array}$ & 762 & 253 & 27.2 & 7.11 & $\mathrm{~A}$ \\
\hline PeGATA2 & PH01000036G1110 & $\begin{array}{l}\text { 651571-655167 } \\
\text { (+ strand) }\end{array}$ & 1212 & 403 & 42.4 & 5.2 & $\mathrm{~A}$ \\
\hline PeGATA3 & PH01000040G1560 & $\begin{array}{l}1013911-1015300 \\
\text { (- strand) }\end{array}$ & 801 & 266 & 28.7 & 9.77 & B \\
\hline PeGATA4 & PH01000114G0660 & $\begin{array}{l}460195-464981 \\
\text { (+ strand) }\end{array}$ & 912 & 303 & 32.2 & 5.96 & $\mathrm{C}$ \\
\hline PeGATA5 & PH01000157G0800 & $\begin{array}{l}521887-523791 \\
(- \text { strand })\end{array}$ & 654 & 217 & 23.2 & 6.15 & A \\
\hline PeGATA6 & PH01000162G1360 & $\begin{array}{l}\text { 945504-954318 } \\
\text { (+ strand) }\end{array}$ & 1500 & 499 & 56.4 & 9.4 & A \\
\hline PeGATA7 & PH01000232G0180 & $\begin{array}{l}85809-87165 \\
(- \text { strand })\end{array}$ & 420 & 139 & 15.6 & 9.23 & B \\
\hline PeGATA8 & PH01000242G0460 & $\begin{array}{l}296415-297844 \\
(- \text { strand })\end{array}$ & 648 & 215 & 22.6 & 8.2 & $\mathrm{~B}$ \\
\hline PeGATA9 & PH01000263G0760 & $\begin{array}{l}473691-475362 \\
\text { (- strand) }\end{array}$ & 1095 & 364 & 37.5 & 7.72 & A \\
\hline PeGATA10 & PH01000284G0590 & $\begin{array}{l}365850-367843 \\
(- \text { strand })\end{array}$ & 1131 & 376 & 39.3 & 5.89 & A \\
\hline PeGATA11 & PH01000417G1130 & $\begin{array}{l}669097-674119 \\
(- \text { strand })\end{array}$ & 948 & 315 & 35.8 & 8.87 & $\mathrm{~A}$ \\
\hline PeGATA12 & PH01000468G1050 & $\begin{array}{l}\text { 681872-683301 } \\
\text { (+ strand) }\end{array}$ & 663 & 220 & 22.7 & 5.78 & $\mathrm{~A}$ \\
\hline PeGATA13 & PH01000604G0620 & $\begin{array}{l}351426-352613 \\
\text { (+ strand) }\end{array}$ & 399 & 132 & 14.6 & 9.36 & A \\
\hline PeGATA14 & PH01000750G0690 & $\begin{array}{l}435897-442169 \\
\text { (+ strand) }\end{array}$ & 777 & 258 & 28.1 & 8.18 & $\mathrm{C}$ \\
\hline PeGATA15 & PH01000836G0660 & $\begin{array}{l}444348-452795 \\
\text { (- strand) }\end{array}$ & 1413 & 470 & 51.6 & 8.57 & C \\
\hline PeGATA16 & PH01000985G0260 & $\begin{array}{l}\text { 141878-143100 } \\
\text { (+ strand) }\end{array}$ & 402 & 133 & 14.8 & 9.87 & $\mathrm{~B}$ \\
\hline PeGATA17 & PH01001002G0190 & $\begin{array}{l}\text { 175975-177694 } \\
\text { (+ strand) }\end{array}$ & 831 & 276 & 29.4 & 8.98 & B \\
\hline PeGATA18 & PH01001129G0380 & $\begin{array}{l}\text { 296297-297943 } \\
\text { (+ strand) }\end{array}$ & 699 & 232 & 26 & 9.16 & B \\
\hline PeGATA19 & PH01001155G0480 & $\begin{array}{l}343290-344746 \\
\text { (- strand) }\end{array}$ & 741 & 246 & 25.3 & 9.66 & $\mathrm{~A}$ \\
\hline PeGATA20 & PH01001253G0390 & $\begin{array}{l}263024-267886 \\
(- \text { strand })\end{array}$ & 516 & 171 & 18.9 & 9.95 & $\mathrm{C}$ \\
\hline
\end{tabular}


Table 1. Cont.

\begin{tabular}{|c|c|c|c|c|c|c|c|}
\hline Name & Gene ID & Location & ORF Length (bp) & Size (aa) & MW (kDa) & PI & Group \\
\hline PeGATA21 & PH01001451G0450 & $\begin{array}{l}\text { 270274-272851 } \\
\text { (- strand) }\end{array}$ & 930 & 309 & 32.6 & 8.68 & $\mathrm{~A}$ \\
\hline PeGATA22 & PH01001557G0370 & $\begin{array}{l}244289-248001 \\
\text { (+ strand) }\end{array}$ & 594 & 197 & 20.7 & 9.14 & $\mathrm{C}$ \\
\hline PeGATA23 & PH01001584G0350 & $\begin{array}{l}297120-303918 \\
\text { (- strand) }\end{array}$ & 1035 & 344 & 37.8 & 4.75 & C \\
\hline PeGATA24 & PH01001907G0160 & $\begin{array}{l}\text { 109783-111580 } \\
\text { (+ strand) }\end{array}$ & 1017 & 338 & 36.2 & 9.26 & B \\
\hline PeGATA25 & PH01002105G0190 & $\begin{array}{l}\text { 151795-153666 } \\
\text { (+ strand) }\end{array}$ & 1248 & 415 & 44 & 8.6 & A \\
\hline PeGATA26 & PH01002473G0050 & $\begin{array}{l}19960-21655 \\
\text { (- strand) }\end{array}$ & 1011 & 336 & 36.1 & 9.64 & B \\
\hline PeGATA27 & PH01002681G0110 & $\begin{array}{l}59436-60698 \\
(- \text { strand })\end{array}$ & 690 & 229 & 24 & 8.49 & B \\
\hline PeGATA28 & PH01002830G0260 & $\begin{array}{l}\text { 174984-177126 } \\
\text { (- strand) }\end{array}$ & 381 & 126 & 13.8 & 9.69 & B \\
\hline PeGATA29 & PH01003365G0100 & $\begin{array}{l}\text { 53196-55342 } \\
\text { (+ strand) }\end{array}$ & 369 & 122 & 13.3 & 9.4 & B \\
\hline PeGATA30 & PH01003433G0110 & $\begin{array}{l}\text { 88789-92096 } \\
\text { (- strand) }\end{array}$ & 1167 & 388 & 39.8 & 9.33 & A \\
\hline PeGATA31 & PH01004789G0060 & $\begin{array}{l}\text { 58264-61570 } \\
\text { (- strand) }\end{array}$ & 993 & 330 & 35 & 6.06 & A \\
\hline
\end{tabular}

bp: base pair, aa: amino acids, MW: molecular weight, PI: isoelectric point, kDa: kilodalton, ORF: open reading frame.

The length of CDS ranged from $366 \mathrm{bp}$ to $1500 \mathrm{bp}$, and the length of proteins ranged from $122 \mathrm{aa}$ to 499 aa (Table 1). PeGATA29 was the smallest GATA protein with 122 amino acids, and the largest protein was PeGATA6 with 499 amino acids (Table 1). The predicted molecular weight of 31 PeGATA proteins ranged from $13.3 \mathrm{kDa}$ (PeGATA29) to $56.4 \mathrm{kDa}$ (PeGATA6) with an average size of $29.86 \mathrm{kDa}$ (Table 1). The predicted PI of 31 PeGATA factors were all below 10.0, and the minimal protein was PeGATA23 with only 4.75 (Table 1).

\subsection{Phylogenetic Analysis of Bamboo GATA Factors}

Phylogenetic analysis with 90 GATA factor sequences from three species (Arabidopsis, rice, and bamboo) showed that these GATA factors were classified into four groups (group A-D, Figure 1). Group A was the largest clade and contained 38 members. In this group, twelve bamboo GATA factors (PeGATA1, -2, -5, -6, -9, -10, -11, -19, -21, -25, -30, and -31) were clustered with 12 rice and 14 Arabidopsis GATA factors (Figure 1). Group B formed the second largest clade, containing 33 members, 13 of which were bamboo GATA factors (PeGATA3, -7, -8, -12, -13,-16, -17, -18, -24, -26, -27, -28, and -29), as well as 9 rice and 11 Arabidopsis GATA factors (Figure 1). Interestingly, six bamboo (PeGATA4, -14, -15, -20, -22, and -23) and seven rice GATA factors were identified in group $C$, while only three were identified from Arabidopsis (Figure 1). Specifically, it is worth noting that no bamboo GATA factor was found in group D (Figure 1), which indicates that bamboo might lack the functions of group D GATA factors compared with Arabidopsis and rice. Consistently, Moso bamboo GATA factors did not cluster with Brachypodium distachyon and soybean group D GATA factors, as shown in Figure S1. 


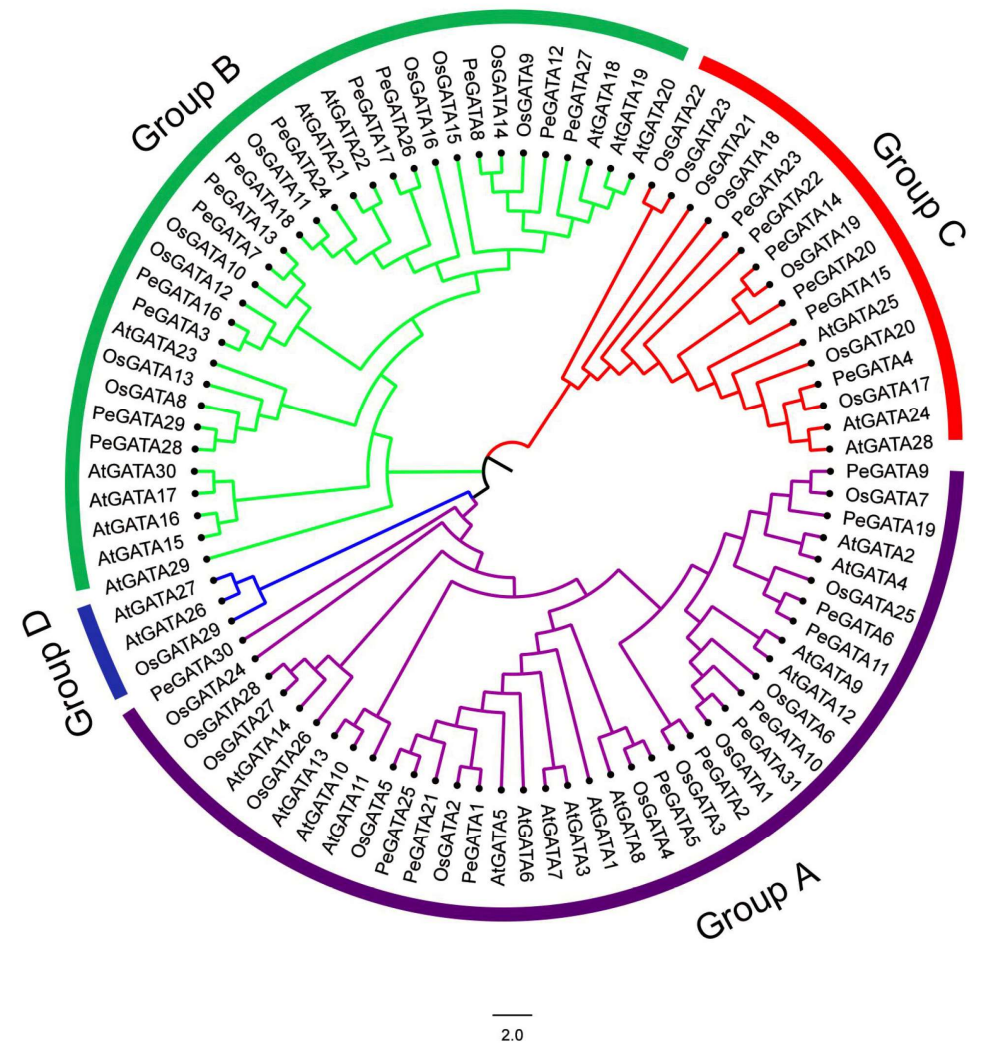

Figure 1. Phylogenetic analysis of GATA factors in bamboo, rice, and Arabidopsis. The phylogenetic tree was made based on the amino acid sequences using MEGA7.0 by the neighbor-joining method with 1000 bootstrap replicates. The tree shows four major phylogenetic groups (A to D) indicated by different colors. The gene names of Arabidopsis, rice and bamboo GATA factors were begun with " $A t$ ", "Os", and " $P e^{\prime}$, respectively. The exact gene IDs of Arabidopsis and rice GATA genes were listed separately in Tables S3 and S4.

\subsection{The Conservation of GATA Motifs in Bamboo GATA Factors}

To further investigate and characterize sequence conservation in the bamboo GATA proteins, multiple sequence alignments were performed using the amino acid sequences of the conserved GATA motifs in 31 PeGATAs (Figure 2). Similar to Arabidopsis and rice, moso bamboo did not contain the animal- and fungal-type $\mathrm{CX}_{2} \mathrm{CX}_{17} \mathrm{CX}_{2} \mathrm{C}$ zinc finger domains (Figure 2). Twenty-six bamboo GATA factors contained 18 residues in the zinc finger loop $\left(\mathrm{CX}_{2} \mathrm{CX}_{18} \mathrm{CX}_{2} \mathrm{C}\right)$, while the other five bamboo GATAs had 20 residues in the zinc finger loop $\left(\mathrm{CX}_{2} \mathrm{CX}_{20} \mathrm{CX}_{2} \mathrm{C}\right)$ (Figure 2). These five bamboo GATA factors were from group $\mathrm{C}$ (Figure 2). It has been reported that rice OsGATA24 contains a partial GATA motif [9]. Interestingly, five bamboo GATA factors (PeGATA1, -14, -17, -18, and -30) had a defective GATA zinc finger domain (Figure 2), and the number of partial GATA motif containing factors was larger in bamboo (5) than in rice (1). The results indicated that most of the bamboo GATA factors had a highly conserved GATA motif.

To further reveal the diversification of GATA factors in moso bamboo, putative conserved functional domains and motifs were also predicted in the NCBI conserved domain database and the MEME (Multiple EM for Motif Elicitation) program. As expected, all of the bamboo GATA factors contain at least one of the GATA motifs (ZnF_GATA, ZnF_GATA superfamily, and GATA, Figure 3A). However, similar to rice but unlike Arabidopsis, three bamboo GATA factors contained two zinc finger domains (Figure 3A), while rice OsGATA24 even contains three and a half GATA motifs [9]. 


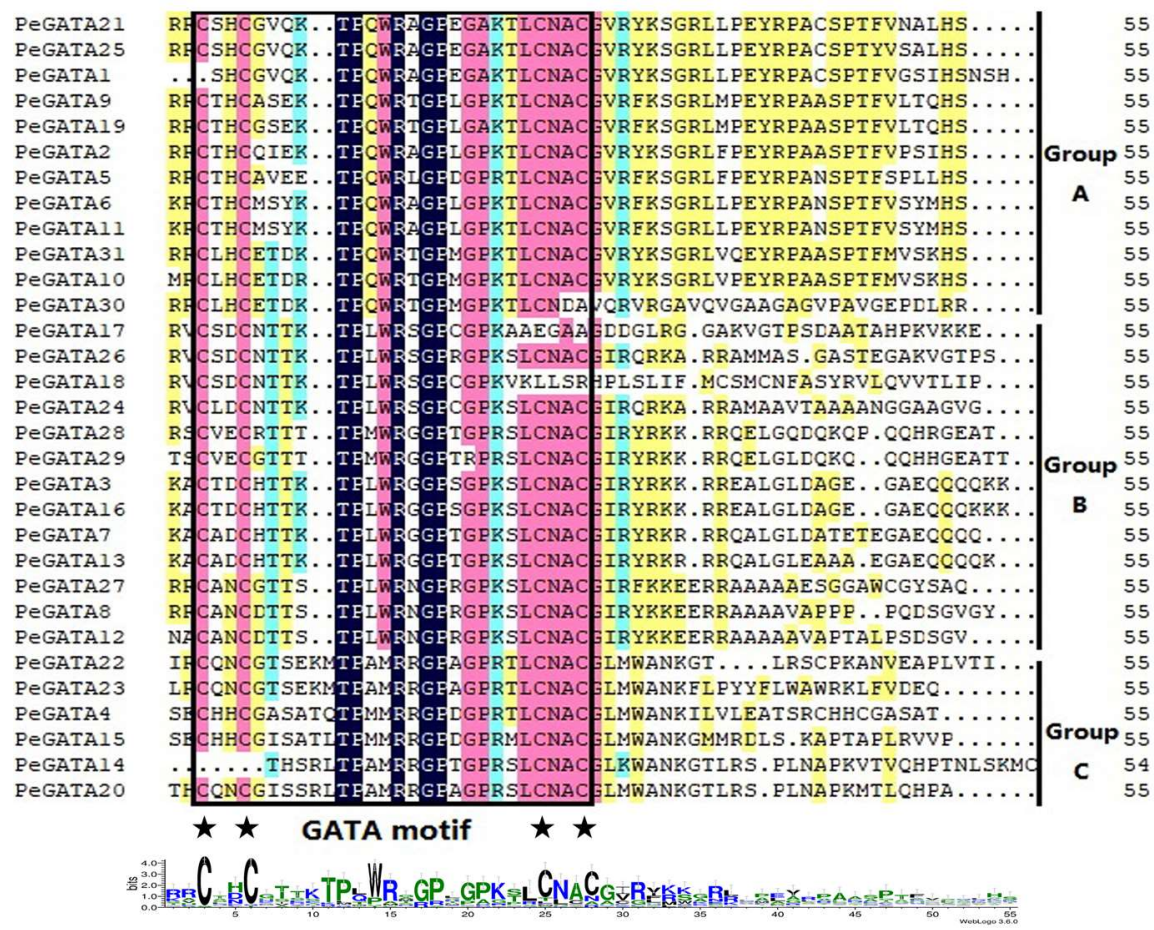

Figure 2. Alignment of the amino acid sequences of bamboo GATA factors. The GATA motif and amino acid positions are marked with a box and an asterisk. The sequence logo of the GATA motif is shown at the bottom.

A

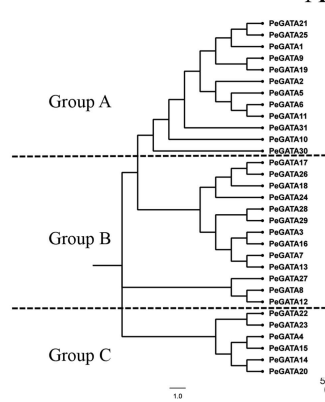

B

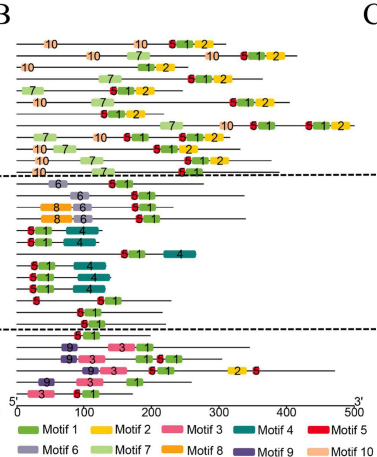

C

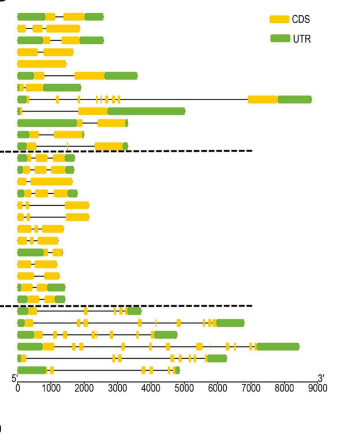

Figure 3. Schematic representation of conserved domains, motifs and gene structure of PeGATA proteins. (A) Conserved domains of PeGATA proteins. (B) Distribution of conserved motifs in PeGATA proteins identified by the MEME. (C) Exon/UTR structures of PeGATA genes. Phylogenetic tree construction of the PeGATA proteins based on the amino acid sequences using MEGA7.0. Each color represents a different domain, motif, and structure of the protein. The position of the sequence and the size of exons or UTRs can be estimated by the scale at the bottom.

Next, through MEME analysis, 10 motifs among the different groups are shown in Figure 3B and the multilevel consensus sequences for these motifs are shown in Table S5. Motif 1 and motif 5 presented in 29 PeGATA proteins and they were annotated as conserved GATA zinc fingers (Figure 3B). However, the other motifs were presented in a group specific manner. For example, three motifs (motif 2, -7, and -10) were exclusively identified in all group A GATA factors except that motif 2 was also identified in PeGATA15 from group C (Figure 3B). Motifs 4, -6 , and -8 were only presented in group B GATA factors (Figure 3B). Motif 3 and motif 9 were only predicted from group C GATA factors (Figure 3B). The identification of group-specific motifs from bamboo GATA factors suggests that these motifs might contribute to the functional differences between the bamboo GATA groups. 


\subsection{Gene Structure of Bamboo GATA Genes}

The gene structure of the PeGATA factors is shown in Figure $3 \mathrm{C}$ and the total exon numbers of PeGATAs from each group were calculated. Group A and group B GATA factors contained two or three exons except PeGATA19 (one exon) and PeGATA6 (nine exons) from group A, while group C GATA factors had five to twelve exons (Figure $3 \mathrm{C}$ ). The gene structure of GATA genes was similar to that of rice [9].

\subsection{Subcellular Localization of Bamboo GATA Factors}

Transcription factors are typically located in the nucleus and regulate transcription of the target genes by binding to the cis-elements in their promoters. Consistent with our hypothesis, subcellular localization assays in tobacco showed that bamboo GATA factors (PeGATA7, -20, -26, and -28) were localized in the nucleus according to the GFP and DAPI stain signals (Figure 4). Localization analysis revealed that bamboo GATA factors might localize in the nucleus to regulate gene expression.

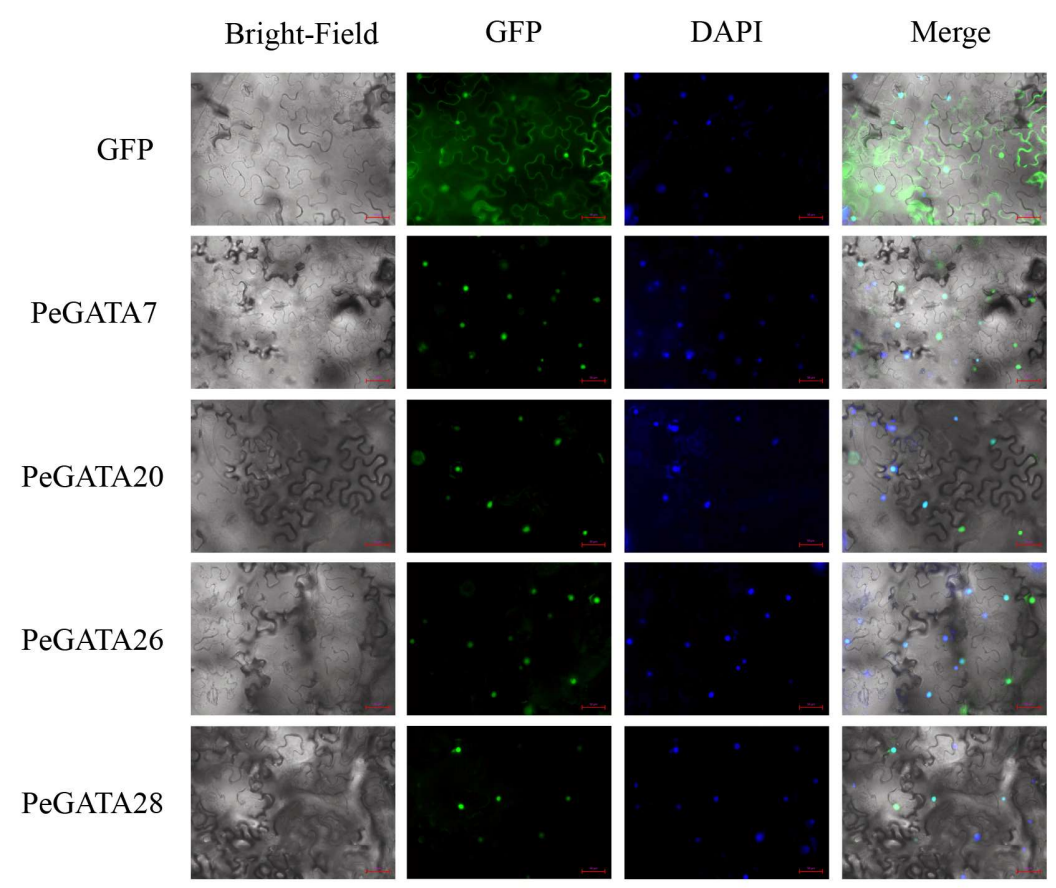

Figure 4. Subcellular localization analyses of bamboo GATA factors. The bamboo GATA genes were cloned and constructed in a modified pCAMBIA3301 vector with a C-terminal GFP fusion. These vectors were transformed into tobacco, and the GFP and DAPI signals were captured from the identical areas by microscopy $(20 \times)$. The red colored scale-bar represents $50 \mu \mathrm{m}$.

\subsection{The Regulatory Cis-Elements in the Promoters of Bamboo GATA Genes}

To further explore the function and regulatory pattern of the PeGATA genes, the PlantCARE database was used to scan the putative cis-elements inside $1500 \mathrm{bp}$ upstream of the translation start site. Light response, development, hormone, and stress-related cis-elements were identified from the promoters of bamboo GATA genes (Figure 5A). Light responsive elements like G-box, GT1, and TCT were widely presented in the promoter of PeGATA genes (Figure 5A), and the G-box element has been reported to be involved in the regulation of chlorophyll II biosynthesis in Arabidopsis [33].

Next, we further analyzed the hormone responsive elements in bamboo GATA promoters. The most dominant hormone responsive element in bamboo GATAs is ABRE for recognizing ABA signal and they were identified in the promoters of 27 bamboo GATAs (Figure 5B). The second abundant cis-elements are related to MeJA and 22 bamboo GATAs have them in their promoter regions (Figure 5B). Interestingly, more GA responsive cis-elements were identified in bamboo GATA promoters than auxin-related 
cis-elements (Figure 5B). Furthermore, the GA-responsive elements were enriched in the group B bamboo GATA factor encoding genes (Figure 5B). Overall, cis-elements analyses indicated that bamboo GATA factors might be involved in response to light and phytohormones to regulate growth.

A

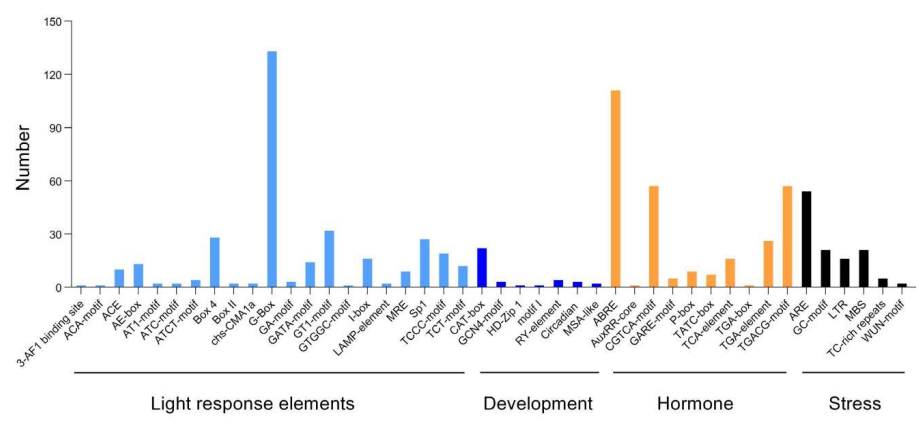

$\mathrm{B}$

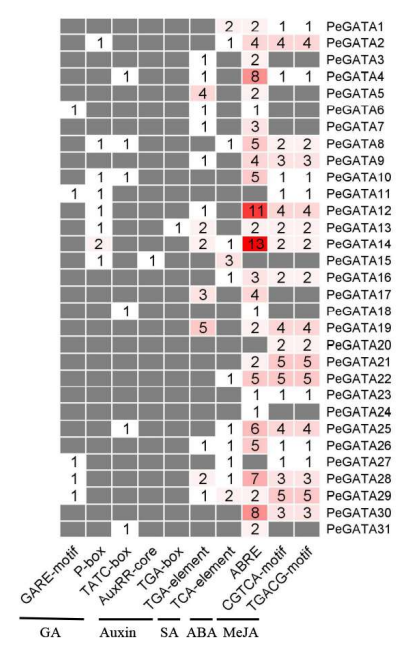

Figure 5. Cis-elements analysis in the promoter of bamboo GATA genes. (A) Overview of the main types of cis-elements identified from the $1.5-\mathrm{kb}$ upstream sequence of the bamboo GATA genes in the PLANTCARE database. (B) Hormone-related cis-elements were analyzed and each colored block with numbers represents the number of cis-elements in the bamboo GATA promoter.

\subsection{Gene Expression Analysis of Bamboo GATAs under Exogenous Hormone Treatment}

GATA factors are closely related to phytohormones to regulate Arabidopsis growth and development $[21,23]$. Taken together with the identification of phytohormone-related cis-elements in the promoter of the bamboo GATA genes (Figure 5B), we rationally hypothesized that the PeGATA genes are also tightly regulated by phytohormones. To test our hypothesis, we performed gene expression analysis of the PeGATA genes under GA, auxin, and ABA treatment based on the published RNA-seq data or qRT-PCR results [28,34].

GA acts as one of central regulators in bamboo internode elongation in both seedling and rapid-growth shoot $[27,28]$. The gene expression analysis of PeGATA genes in bamboo seedlings under exogenous GA treatment was performed in a previous study [28]. Briefly, 4-week-old moso bamboo seedlings were treated with or without $\mathrm{GA}_{3}(100 \mu \mathrm{M})$ for $4 \mathrm{~h}$ and samples were collected for constructing RNA-seq libraries [28]. The RNA-seq data was mapped with Tophat2 and the expression of bamboo genes was normalized based on fragments per kilobase of transcript per million mapped reads (FPKM) [28]. The normalized expression of PeGATA genes was extracted from the published GEOdataset (GSE104596) [28]. A total of 10 PeGATA genes (PeGATA4, -8, -9, -10, -14, -15, -19, -30, and -31) showed increased gene expression in $\mathrm{GA}_{3}(100 \mu \mathrm{M})$ treated seedlings compared to that from untreated control (Figure 6A, Table S6). On the contrary, the expression of nine PeGATA genes (PeGATA1, -6, -16, -17, -18,-21, -24, -25, and -26) was reduced under GA treatment. PeGATA26 was the most down-regulated gene with a 56\% expression level reduction, followed by PeGATA18 with a 54\% decline. The GA responsive elements were also identified in $10 \mathrm{GA}$ associated bamboo GATA genes (PeGATA4, -6, -8, -10,-14,-15,-18,-19, -25, and -31). These results indicate that GA might regulate the gene expression of PeGATAs.

Auxin plays important roles in bamboo shoot rapid-growth and seedling development $[27,34]$. To test the relationship between PeGATA gene expression and auxin, we analyzed the gene expression pattern of PeGATA genes under NAA treatment ( $5 \mu \mathrm{M}$ NAA) in bamboo seedlings, according to the previously published RNA-seq data [34]. Briefly, one-month-old moso bamboo seedlings were treated 
with or without NAA $(5 \mu \mathrm{M})$ for $4 \mathrm{~h}$ [34]. The roots of these samples were used for an RNA-seq experiment [34], and the RNA-seq data were analyzed exactly the same as the GA experiments [28]. The normalized expression of PeGATA genes was extracted from the GEOdataset (GSE100172). Interestingly, unlike GA treatment, the gene expression of most PeGATA genes did not change under auxin treatment (Figure 6B, Table S7). Under auxin treatment, the expression of three PeGATA genes (PeGATA9, -29, and -28) was decreased, while the expression of two PeGATA genes (PeGATA8 and PeGATA15) was promoted. Moreover, all of these genes except PeGATA8 contained the auxin responsive cis-elements. Overall, our result suggested that auxin might have a limited effect on the expression regulation of bamboo GATA genes.

A

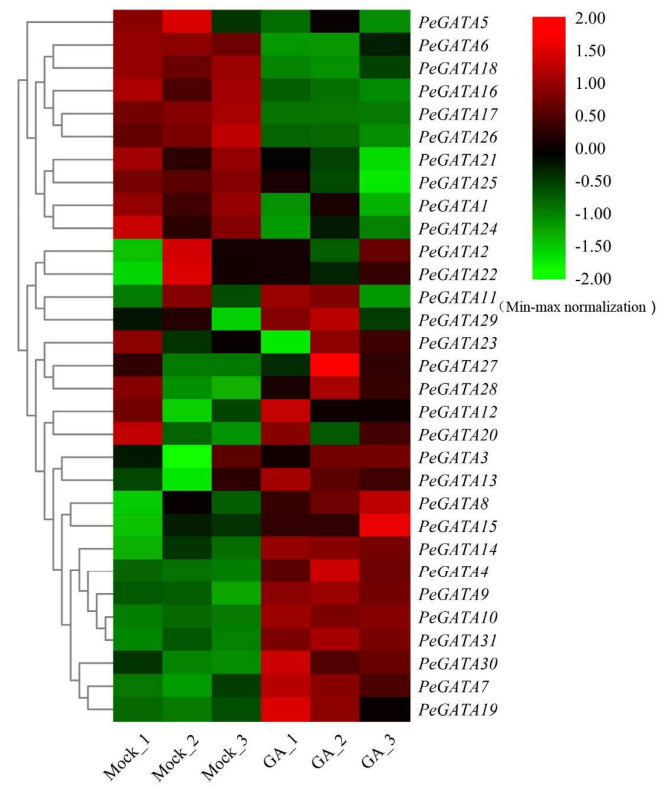

B

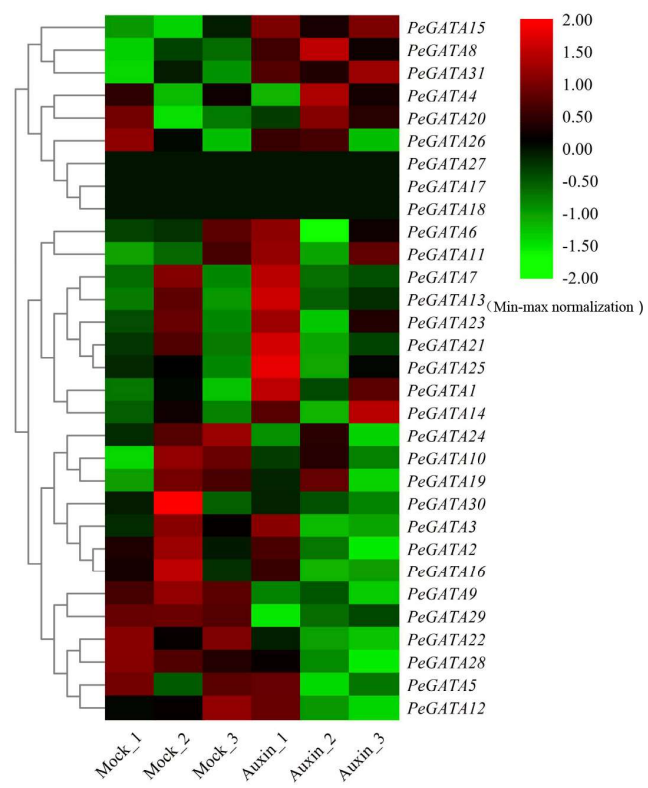

Figure 6. Expression profiles of moso bamboo GATA genes under GA and auxin treatments. (A) The heatmap of PeGATAs gene expression level in moso bamboo seedlings under GA treatment. (B) The heatmap of PeGATAs gene expression level in moso bamboo seedlings under auxin treatment. The FPKM (Fragments per Kilobase Million) value of PeGATA genes were normalized by the min-max method, according to the rows, and presented at the right side. Green represents a low level and red indicates a high level of transcript abundances.

To test the relationship between PeGATA gene expression and ABA, we analyzed the gene expression pattern of all $31 \mathrm{PeGATA}$ genes under a time-course ABA treatment (control, $1 \mathrm{~h}, 6 \mathrm{~h}$, and $24 \mathrm{~h}$ ) in bamboo seedlings by qRT-PCR. Twenty-one of them (PeGATA2, -3, -5, -6, -8, -9, -11, -12, -13, $-17,-18,-19,-20,-22,-23,-24,-25,-26,-28,-30$, and -31 , Figure 7) changed their expression pattern in one of the ABA time-course treatment stages (Figure 7). ABA treatment increased the expression of fifteen bamboo GATA genes (PeGATA2, -3, -5, -8, -9, -13, -17, -19, -22, -23, -24, -25, -28, -30, and -31, Figure 7). Especially, the expression of PeGATA31 was highly induced by ABA (Figure 7). Interestingly, only five tested bamboo GATA genes (PeGATA6, -11,-12,-18, and -20) showed reduced expression under ABA treatment (Figure 7). Among the 21 ABA-related bamboo GATA genes, all of them, except PeGATA11 and PeGATA22, contain the ABA responsive cis-elements in their promoters. Overall, our results suggested that $\mathrm{ABA}$ might induce the expression of bamboo GATA genes. 

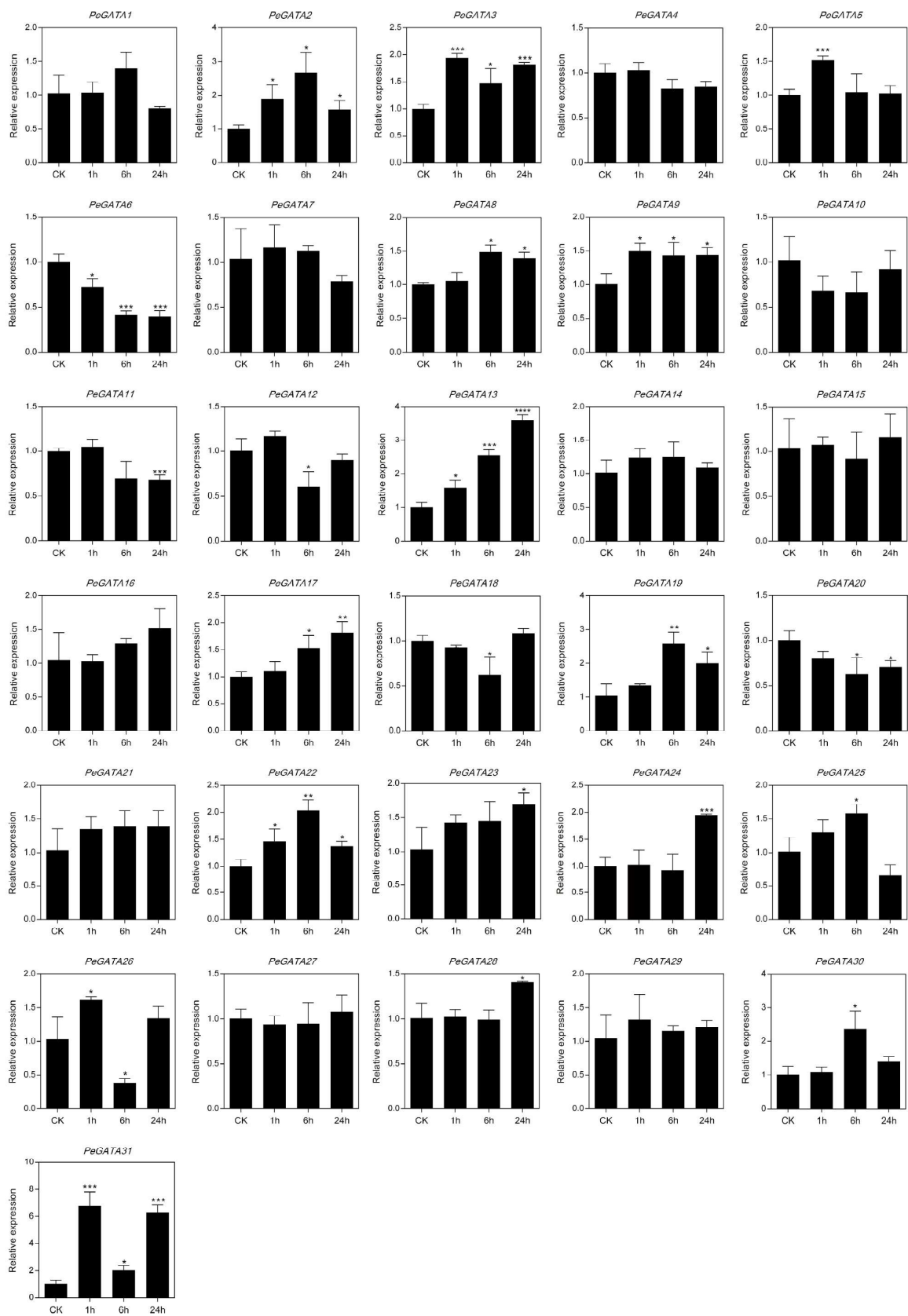

Figure 7. qRT-PCR expression analysis of PeGATA genes following abscisic acid (ABA) treatment. Untreated sample was set as control (CK) and its expression levels were normalized to 1 . Bars indicate standard deviations (SD) from three biological replicates and asterisks indicate a significant difference between the treatment and CK groups $\left({ }^{*} p<0.05,{ }^{* *} p<0.01,{ }^{* * *} p<0.001\right)$.

\subsection{Gene Expression Analysis of Bamboo GATA Genes during the Rapid-Growth of Bamboo Shoots}

As the rapid-growth of bamboo shoots is largely determined by phytohormone and nutrients [2,27], and we have demonstrated that PeGATAs were differentially expressed under different phytohormone treatments (Figures 6 and 7), we hypothesized that PeGATAs might also involve in the fast-growth of bamboo shoots. To validate this hypothesis, the expression profiles of the PeGATAs in the fast-growing bamboo shoots $(0.15,0.5,1.6,4.2$ and $9 \mathrm{~m})$ were examined by qRT-PCR (Figure 8). Interestingly, most of PeGATA genes had a lower expression during the rapid-growth of bamboo shoots (Figure 8). Especially, nine PeGATA genes decreased their expression of rapid-growth of bamboo (PeGATA5, -6, -11, -12, -16, $-21,-22,-26$, and -31 , Figure 8). Five of them (PeGATA6, -16, -21, -26, and -31) were responsive to GA 
treatment, and all of them, except PeGATA31, also decreased their expression under GA treatment (Figure 6A). The results indicated that these genes might negatively correlate with the rapid-growth of shoots probably via GA signaling pathway. Other sets of PeGATA genes (PeGATA3, -4, -7, -9, -10, -15 , and -24) did not have change in expression at the $0.5 \mathrm{~m}$ shoot developmental stage, but reduced expression at other rapid-growth stages (Figure 8). Five of them were in response to GA treatment and all of them increased expression under GA treatment except PeGATA24 (Figure 6A). The results suggest that these genes might have function at the $0.5 \mathrm{~m}$ shoot development stage and GA might also regulate their expression. In addition, three PeGATA genes (PeGATA2, -17, and -18) have increased their expression at the $0.5 \mathrm{~m}$ developmental stage. Interestingly, we did not find that any PeGATA genes continued to increase their expression along with bamboo shoot development. Overall, our results indicated that PeGATAs genes might be involved in the rapid-growth regulation of bamboo shoots.

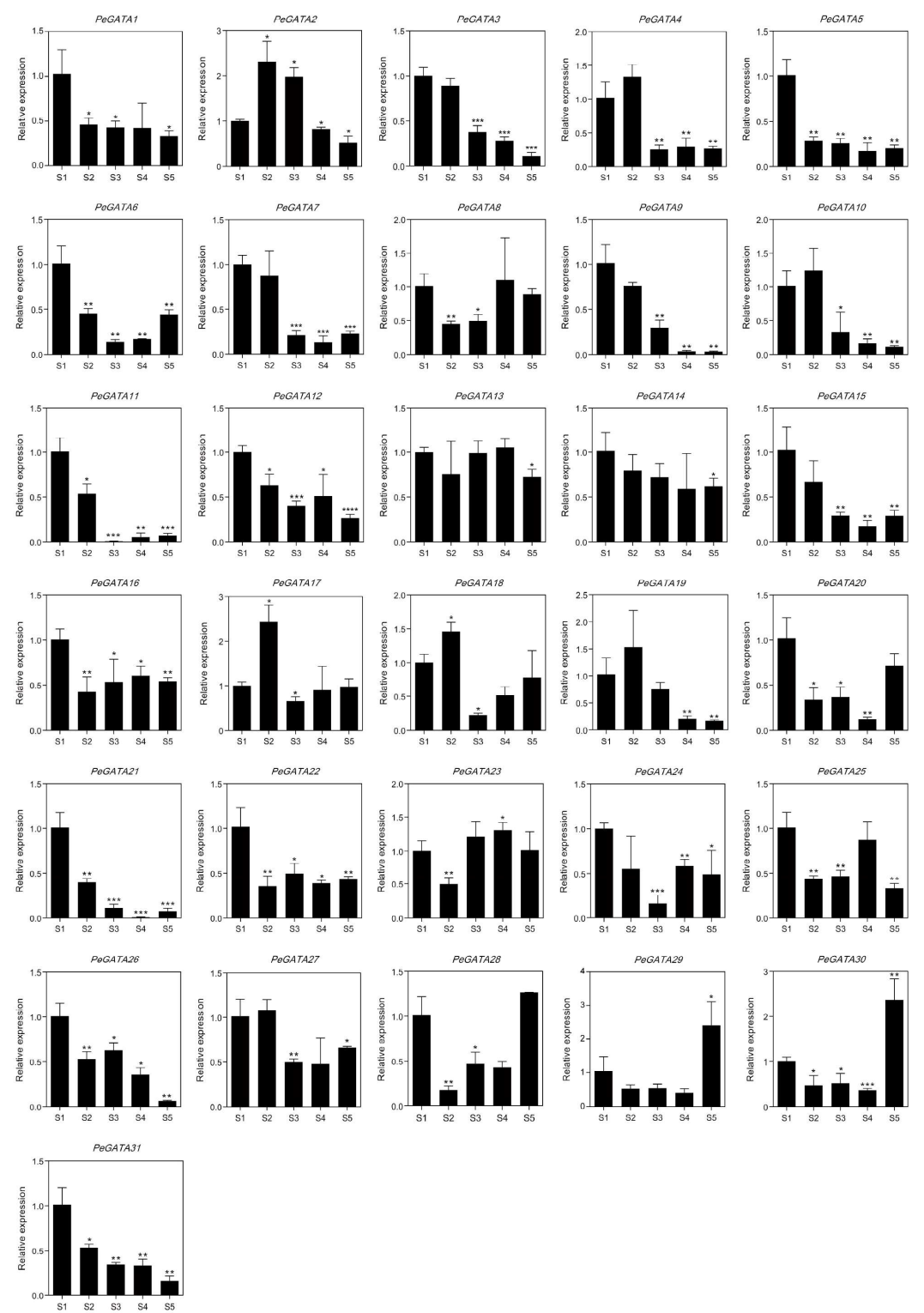

Figure 8. qRT-PCR expression analysis of PeGATA genes in bamboo shoots. The $y$-axis and $x$-axis indicate relative expression level at different heights of shoots. S1: $0.15 \mathrm{~m}$ shoots; S2: $0.5 \mathrm{~m}$ shoots; S3: $1.6 \mathrm{~m}$ shoots; S4: $4.2 \mathrm{~m}$ shoots; S5: $9 \mathrm{~m}$ shoots. Asterisks indicate a significant difference between the higher shoots and the 0.15 m shoots $\left({ }^{*} p<0.05,{ }^{* *} p<0.01,{ }^{* * *} p<0.001\right)$. 


\subsection{Gene Expression Analysis of Bamboo GATAs during Rhizome Development}

The lateral buds, rhizome tips, and new shoot tips are important for rhizome development, and the picture of these tissues has been depicted in previous study [2]. The widespread rhizome system is essential for rapid-growth of bamboo shoots through adopting and utilizing nutrients [35]. By analyzing the RNA-seq data from previous study [2], the expression pattern of PeGATA genes showed a tissue-specific pattern in rhizome (Figure 9, Table S8).

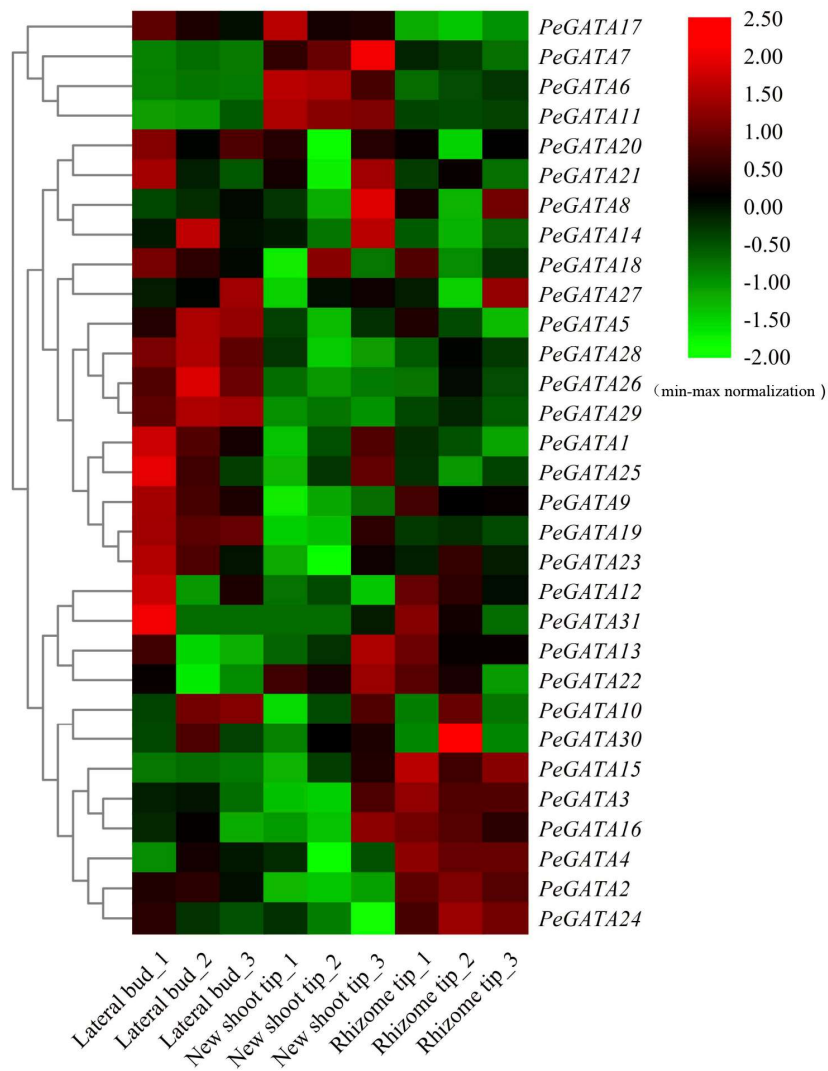

Figure 9. The expression profiles of PeGATA genes in different rhizome tissues. Expression values were normalized and presented at the right side, green represents a low level and red indicates a high level of transcript abundances. Each tissue has three replicates.

As shown in Figure 9, thirteen bamboo GATA genes might play important roles in the development of different rhizome tissues. PeGATA17 exclusively reduced expression in rhizome tips, while five PeGATAs (PeGATA2, -3, -4, -15, and -24) increased expression more in rhizome tips than in the other two tissues (Figure 9). More importantly, among them, four PeGATAs (PeGATA4, -15, -17, and -24) were responsive to GA and all of them, except PeGATA24, increased expression under GA treatment (Figure 9). Therefore, the results suggest that these PeGATA genes might play important roles in controlling the development of rhizome tips and the phytohormone GA might also be involved in the regulation expression of these genes. Next, the expression of three PeGATA genes (PeGATA6, -7, and -11) were exclusively increased in new shoot tips of the bamboo rhizome, and only PeGATA6 had an opposite expression pattern, from GA treatment. The results suggest that these three PeGATA genes might play central roles in new shoot development. Finally, eight PeGATA genes (PeGATA1, -9, -19, -20, $-23,-26,-28$, and -29) were identified to have an increased expression in the lateral bud compared to the other tissues (Figure 9). Interestingly, three of them (PeGATA9, -28, and -29) were responsive to auxin treatment (Figure 6B). More importantly, all of them have an opposite expression pattern between the lateral bud and auxin treatment (Figure 6B). Therefore, the result indicates that these four GATA genes might be involved in the growth regulation of lateral buds and probably via the auxin pathway. 


\subsection{PeGATA26 May Negatively Regulate Plant Height in Arabidopsis}

To understand the function of PeGATA factors, PeGATA26 was chosen as an example to verify the role of PeGATA factors in plant growth. GA, ABA, and MeJA-related cis-elements were also identified in the PeGATA26 promoter (Figure 5). PeGATA26 showed higher gene expression in the growth-inactive lateral buds than the growth-active rhizome tips and new shoot tips (Figure 9). Moreover, PeGATA26 have lower gene expression under GA treatment (Figure 6A), and its expression decreased in rapid-growth bamboo shoots (Figure 8). These results suggest that PeGATA26 might act as a negative regulator of plant growth and height in moso bamboo. Therefore, we hypothesized that PeGATA26 plays a crucial role in regulating plant growth. In a previous study, we successfully characterized one of the fast growth-suppressing genes PeGSK1 by over-expressing it in Arabidopsis [1]. Therefore, we used a similar strategy to verify the function of PeGATA26 in regulating plant growth by over-expressing it in Arabidopsis.

The homozygous $\mathrm{T} 3$ transgenic lines were used to analyze the phenotype, and over-expression of PeGATA26 resulted in a significant growth retardation phenotype (Figure 10A). Gene expression of PeGATA26 was successfully detected by qRT-PCR (Figure 10B). The phenotypes between the two over-expressing lines were similar and the intensity of phenotype correlated with the expression of each of the transgenic lines (Figure 10A). Therefore, PeGATA26 over-expressing line 1 (PeGATA26-ox1), with a stronger phenotype, was used for further phenotypic analysis. Interestingly, PeGATA26-ox1 showed a significant dwarf phenotype with a dramatic shorter inflorescence compared to the control (Figure 10C), indicating that PeGATA26 inhibits growth of plant height. Moreover, PeGATA26 also repressed primary root growth (Figure 10C-D). However, PeGATA26 promoted Arabidopsis hypocotyl length (Figure 10C-D). These results indicate that PeGATA26 may regulate plant growth in a tissue-specific manner-repressing cell growth in roots and inflorescences, while promoting cell growth in hypocotyls.

As PeGATA26 was down-regulated under the GA treatment in bamboo seedlings (Figure 6A), we subsequently analyzed whether PeGATA26 is also regulated by GA in Arabidopsis. Interestingly, exogenous GA treatment did not complement the dwarf phenotype of PeGATA26-ox1 (Figure 10E-F), indicating that PeGATA26 might act downstream of the GA biosynthesis pathway to regulate plant height. Furthermore, qRT-PCR analyses showed that the expression of GA signaling related genes including RGA1, GAI, GID, GID1B, and GID1C did not change in the PeGATA26-ox1 transgenic line. Instead, the GA turnover-related genes GA3 oxidase 1 and GA2 oxidase 2 were significantly increased and reduced, respectively, in the PeGATA26-ox1 transgenic line (Figure 10G). The increased GA3 oxidase 1 and decreased GA2 oxidase 2 expression is correlated with the promoting GA turnover and thus decreases the GA level [21]. Therefore, PeGATA26 might repress Arabidopsis growth via stimulating the GA turnover. Overall, these results suggest that PeGATA26 inhibits the plant root and stem growth in Arabidopsis, and-together with the gene expression-might negatively correlate with the growth of shoots in moso bamboo. We concluded that PeGATA26 might be a negative growth regulator for plant height control from Arabidopsis to moso bamboo, probably via promoting GA turnover. 
A

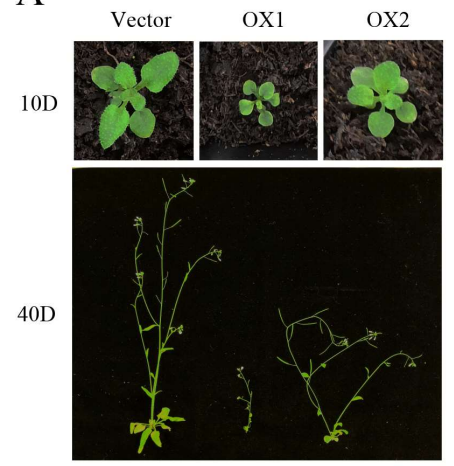

D

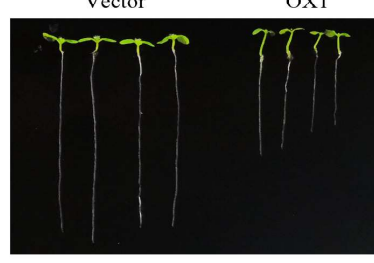

$\mathrm{F}$

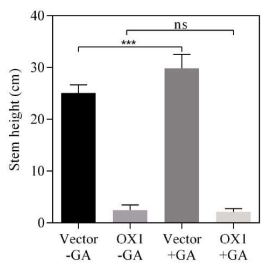

B

$\mathrm{C}$
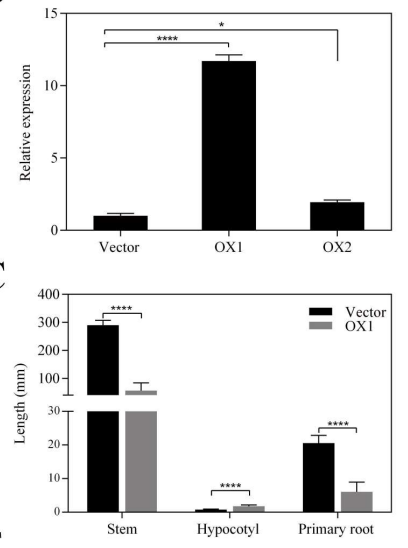

$\mathrm{E}$

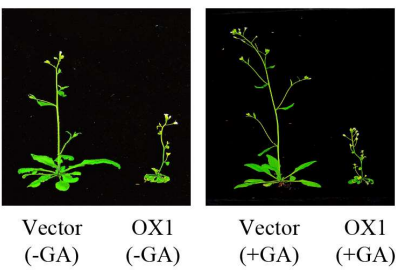

$\mathrm{G}$

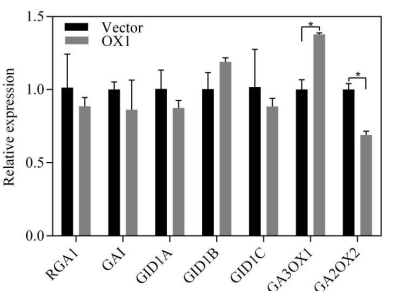

Figure 10. Ectopic expression of PeGATA26 inhibits the plant height of Arabidopsis. (A) Over-expression of PeGATA26 resulted in a dwarf phenotype in Arabidopsis. 10D: 10-day-old seedlings, 40D: 40-day-old seedlings. (B) The expression level of PeGATA26 was detected in both transgenic lines with ACTIN2 gene as internal control. (C) Phenotypic analysis of plant height, hypocotyl length, and primary root length in PeGATA26-ox1 compared with the control (>30 individual plants). (D) Hypocotyl and primary root phenotype of 7-day-old PeGATA26 over-expression line 1 (PeGATA26-ox1). Phenotypic (E) and statistical (F) analyses of PeGATA26-ox1 were resistant to exogenous GA treatment. (G) The expression of GA signaling genes was detected by qRT-PCR, and ACTIN2 was used as an internal control. Asterisks (Student's $\mathrm{t}$ test, ${ }^{*} p<0.05,{ }^{* * *} p<0.001,{ }^{* * * *} p<0.0001$, ns: no significance, $p \geq 0.05$ ) marked significant difference between PeGATA26-ox and control.

\section{Discussion}

Moso bamboo is one of the important non-timber forestry species with great value in providing food and building materials [36]. Moreover, bamboo is known for its fast-growing shoots and widespread rhizomes [2]. It has been reported that several gene families are involved in flower development and abiotic stress [30-32,37]; however, the rapid-growth associated transcription factors remain elusive. The genome sequences of moso bamboo [36] and transcriptome studies [2,27,28,34] provide important platforms for the identification of rapid-growth shoot and rhizome development associated gene families. The rapid-growth related genes could provide useful information for genetic manipulation of plant height in the future.

Bioinformatics analysis showed that there are 31 PeGATA factors in moso bamboo (Table 1). The number of bamboo GATA factors is closer to other species, including Arabidopsis (29), rice (28), and apples $(35)[9,10,15]$. Furthermore, most PeGATA factors have a conserved single $\mathrm{CX}_{2} \mathrm{CX}_{18-20} \mathrm{CX}_{2} \mathrm{C}$ zinc finger domain that is highly similar to that from Arabidopsis and rice (Figure 2) [9]. In addition, the groups of $\mathrm{A}$ to $\mathrm{C}$ from moso bamboo showed a highly evolutionary conservation compared to 
Arabidopsis and rice (Figure 1). These results indicate that most of the GATA factors from moso bamboo are conserved compared to other species. However, unlike containing only one zinc finger domain in group A, GATA factors from Arabidopsis and rice [9], PeGATA6 and PeGATA11 from the bamboo GATA group A, have two GATA-type zinc finger domains (Figure 3A).

Moreover, more protein domains from the bamboo GATA group $C$ were identified compared to that from Arabidopsis and rice (Figure 3). Interestingly, a unique feature of the PeGATA factors is that they only have three groups compared to the four groups of Arabidopsis and rice (Figure 1). These differences suggest that PeGATAs do have certain specificity compared to that from Arabidopsis and rice. Future analysis of the functions of GATA factors, including AtGATA26, AtGATA27, and OsGATA30 from group D (Figure 1), can help us reveal why bamboo lacks these GATA factors.

The first GATA factor is identified according to the light and circadian clock-related cis-elements in its promoters [13]. Moreover, the Arabidopsis GATA factors AtGATA1, AtGATA2, and AtGATA4 have been reported to be involved in light regulation of gene expression and photomorphogenesis [22,38]. Thus, the function of the GATA factors can be predicted based on the identification of cis-elements from their promoter. In this study, we found that the promoter of PeGATAs has many important cis-elements, including light responsive elements, and cell cycle regulation and phytohormone responsive elements (Figure 5), which are closely related to the regulation of plant growth. Thus, PeGATAs may be involved in regulating plant growth through these cis-elements to affect their gene expression and further downstream genes.

Bamboo has a well-established rhizome system to develop new shoot tips and widespread rhizome tips [2,35]. However, the lateral buds of bamboo rhizomes are not active and dominant in growth [2]. Therefore, identification of GATAs with different expression patterns in these tissues will help us clarify the role of GATA factors in rhizome development, which presently remains unclear. In this study, we found that eight PeGATA genes are highly expressed in lateral buds (Figure 9). Among them, the orthologous gene of PeGATA9, AtGATA2 has been reported to have a function to restrict cell division in the proliferation domain of Arabidopsis root meristem [39], and high expression of PeGATA9 in lateral buds indicates that PeGATA9 may also involve in inhibiting the cell division in bamboo lateral buds (Figure 9). In contrast, the lower expression of PeGATA9 in the actively growing new shoot tips and rhizome tips (Figure 9), suggests a negative correlation between PeGATA9 and cell growth in bamboo rhizome.

Researchers reported that AtGATA22, an orthologous gene of PeGATA18, is involved in response to cytokinin and negatively regulates root growth in Arabidopsis [40]. We found that PeGATA18 has higher gene expression in lateral buds (Figure 9), suggesting that PeGATA18 may play a role in negative regulation of cell growth. Overall, these results suggest that these PeGATAs may contribute to negatively regulating cell growth in lateral buds. Next, five PeGATAs were highly expressed in the new shoot tips compared to in the other two tissues (Figure 9). Furthermore, five PeGATAs were highly expressed in the rhizome tips, indicating that they are involved in the growth of the rhizome tips (Figure 9). Once the transformation system is ready in the future or by mutagenesis with EMS, functional characterization of these PeGATAs in moso bamboo will help us elucidate the exact role of PeGATAs in rhizome growth control.

The correlation between GATA factors and GA or auxin has been extensively studied in Arabidopsis [21,23,39,41]. In this study, we found that the gene expression of 19 PeGATAs changed under GA treatment, while only five PeGATAs responded to auxin treatment (Figure 6). In addition, motif analysis indicated that the promoter of PeGATAs has more GA-related cis-elements than auxin (Figure 5B). Our results indicate that GA, rather than auxin, frequently regulates the expression of PeGATAs in moso bamboo.

Gene expression analysis showed that most PeGATAs change their expression during the rapid-growth of bamboo shoots (Figure 8). For example, the expression of PeGATA9 was down-regulated over 30 times in the late rapid-growth stage $(9 \mathrm{~m})$ than the early stage $(0.15 \mathrm{~m})$ (Figure 8$)$. The results indicate that PeGATA9 may negatively regulate the rapid-growth of bamboo shoots. Identification of 
many rapid-growth-related PeGATAs indicates that PeGATAs are involved in regulating the bamboo shoot. The rapid-growth of bamboo shoots is tightly controlled by phytohormones [27]. Current studies reveal that $\mathrm{ABA}$ is the only negative regulator of fast-growing shoots, while BR, auxin, GA, and cytokinin antagonize with ABA to promote rapid-growth of bamboo shoots [27]. Interestingly, all of the 19 GA-related and 21 ABA-related PeGATAs showed differential expression in at least one of the rapid-growth stages (Figures $6 \mathrm{~B}$ and 8 ), suggesting that $\mathrm{GA}$ and $\mathrm{ABA}$ may regulate rapid-growth of bamboo shoots via modulating the gene expression of PeGATAs.

To understand the function of GA-related PeGATAs in plant height control, PeGATA26 was selected to validate its role in Arabidopsis growth (Figure 10). The orthologous gene of PeGATA26 in Arabidopsis is GNC (AT5G56860), which plays negative roles in seed germination, flowering, and leaf elongation growth [21]. Over-expressing GNC repressed Arabidopsis germination, leaf expansion, and flowering [21]. Similarly, over-expression of PeGATA26 in Arabidopsis resulted in growth retardation phenotypes such as dwarfism and shorter primary root length, and the PeGATAs over-expressed lines were resistant to GA treatment (Figure 10). Future study focused on complementing the PeGATA26 into Arabidopsis gnc mutants will help us clarify the functional similarity and difference between PeGATA26 and GNC. Overall, these results further support that PeGATAs could regulate plant heights from Arabidopsis to moso bamboo via downstream of the GA pathway.

\section{Material and Methods}

\subsection{Plant Materials and ABA Treatment}

The shoots of moso bamboo (Phyllostachys edulis, $0.15 \mathrm{~m}, 0.5 \mathrm{~m}, 1.6 \mathrm{~m}, 4.2 \mathrm{~m}$, and $9 \mathrm{~m}$ ) used in this study were collected from wild bamboo forestry with no permission needed in JianOu County $\left(\mathrm{E} 118^{\circ} 28^{\prime} ; \mathrm{N} 27^{\circ} 00^{\prime}\right)$, Fujian Province, China. The moso bamboo plants were authenticated by Zhenguo $\mathrm{Xu}$ (Guangxi Forestry Research Institute, Nanning, Guangxi Province, China) and a voucher specimen has been deposited in Herbarium, Guangxi Forestry Research Institute (voucher No. 2018060325). To ensure the high similarity growth conditions of bamboo shoots, all the shoots were collected in an area of $50 \times 50 \mathrm{~m}^{2}$ [42]. The middle internode of different heights of bamboo shoots, which was determined using an equal division method, were sampled and stored in liquid nitrogen immediately, as recommend by a previous study [43]. For ABA treatment, 4-week-old moso bamboo seedlings grown in soil were transformed to Hoagland solution for an additional one week of cultivation and then treated with $10 \mu \mathrm{M}$ ABA (CAISSON, A036-100MG, Smithfield, UT, USA) in a time-course experiment $(0,1,6$, and $24 \mathrm{~h})$. Samples were harvested and RNA was isolated to perform future expression analysis.

\subsection{Identification of GATA Factors in Moso Bamboo}

To identify the GATA factors, the genome and protein sequences of moso bamboo were downloaded from BambooGDB database (http://forestry.fafu.edu.cn/db/PhePacBio/download.php) [36]. GATA protein sequences from Arabidopsis and rice were obtained from previous published data [9]. We performed multiple sequence BLAST and alignment with an expected value of 10. The HMMER profile of the GATA domain (PF00320) from Pfam (http://pfam.xfam.org/) was used to search the bamboo protein database with a threshold: $e$-values $<10^{-5}$ [44]. The bamboo GATA factors were determined with the criteria: Present in both BLAST and HMMER motif analysis lists. The number of amino acid, molecular weights (MWs), and isoelectric points (PI) of bamboo GATA factors were predicted by ProtParam (https://web.expasy.org/protparam/).

\subsection{Phylogenetic Tree, Conserved Domain, Motif Recognition and Cis-Elements Analyses}

Multi-sequence alignment of the GATA protein sequences was carried out by ClustalX [45], and phylogenetic tree was constructed using MEGA7 by the Neighbor-Joining method (bootstrap analysis for 1000 replicates) [46]. Conserved domains were obtained from NCBI (https://www.ncbi.nlm. nih.gov/cdd) [47] and motifs were analyzed using MEME with default parameters (version 5.0.5, 
http://meme-suite.org/tools/meme) [48]. For cis-elements analysis, DNA sequences from $1.5 \mathrm{~kb}$ upstream region of each PeGATA gene were used to scan any potential cis-element using the PlantCARE database (http://bioinformatics.psb.ugent.be/webtools/plantcare/html/) [49].

\subsection{Subcellular Localization Analysis}

To verify the location of PeGATAs, the full-length CDSs without stop codon from four PeGATA genes were cloned into a modified pCAMBIA3301 vector with C-terminal GFP, as described in a previous study [1]. The ACTIN2::PeGATAs-GFP and the ACTIN2::GFP control constructs were then transiently transformed into tobacco as described in a previous study [50]. We used $1 \mathrm{mg} / \mathrm{L} \mathrm{DAPI}$ (SIGMA, D9542, St. Louis, MO, USA) to stain the nucleus for $30 \mathrm{~min}$, and the identical region was observed using a microscope (20×, Zeiss, Axio Observer A1, Jena, Germany) to detect GFP and DAPI fluorescence signals. The two photographs with identical regions from GFP and DAPI were then merged into a new photograph, in which the overlapping of GFP and DAPI signals represented a nuclear localization of bamboo GATA factors.

\subsection{Gene Expression Analysis}

To investigate gene expression levels of the PeGATA genes in different tissues or hormone treatments, RNA-seq data was downloaded from the Short Read Archive (SRA) database for the lateral buds, rhizome tips, and new shoot tips (SRP093919) [2], and bamboo seedlings under GA and auxin treatment (GSE104596 and GSE100172) [28,34], respectively. The pair-end reads were mapped to the moso bamboo reference genome using Tophat 2 , and differentially expressed genes were detected by Cufflinks with default parameters [51].

qRT-PCR analysis was performed for each member of the GATA family genes during the rapid-growth of bamboo shoots or ABA treated seedlings. Total RNA was extracted from the bamboo samples using the HiPure Plant RNA Mini Kit (Magen, R4151-02, Guangzhou, China) and $1 \mu$ g RNA was taken for reverse transcription into cDNA using a commercial Kit (Monad, RN05004M, Suzhou, China). Primers for qRT-PCR were designed on Primer3 (http://primer3.ut.ee/) using the CDS of each PeGATA gene. qRT-PCR were performed using MonAmp ${ }^{\mathrm{TM}}$ ChemoHS qPCR Mix (Monad, RN04002M, Suzhou, China) in a $20 \mu \mathrm{L}$ reaction. The following program was used for qRT-PCR: $95^{\circ} \mathrm{C}$ for $5 \mathrm{~min}$; 40 cycles of $95^{\circ} \mathrm{C}$ for $10 \mathrm{~s}, 60^{\circ} \mathrm{C}$ for $10 \mathrm{~s}$, and $72{ }^{\circ} \mathrm{C}$ for $30 \mathrm{~s}$. For qRT-PCR data analyses under ABA treatment, the expression of PeGATA genes in treated bamboo seedlings was normalized to control condition. For qRT-PCR data analyses for rapid-growth bamboo shoots, the expression of PeGATA genes from the S2-S5 stages was normalized to the expression of the S1 stage. The expression of GA signaling genes in PeGATA26 transgenic lines was normalized according to the expression in empty vector over-expressing control lines.

\subsection{Ectopic Expression Analysis}

PeGATA26 was cloned and expressed in Arabidopsis following exactly the procedures for the PeGSK1 in previous study [1]. The T3 generation seedlings were used for phenotype analysis. Seven-day-old PeGATA26 over-expressing transgenic or empty vector control lines ( $>30$ individual plants) were used to measure the primary root length or hypocotyl length with ImageJ [1], respectively. The height of 40-day-old Arabidopsis plants was measured using a ruler. The $p$-value was calculated by T-test and the number of asterisks represented the extent of significance difference $\left({ }^{*} p<0.05,{ }^{* *} p<0.01,{ }^{* *} p<0.001\right.$, **** $p<0.0001)$.

The primers used in this study are listed in Table S9.

\section{Conclusions}

With the explosive growth rates of bamboo shoots and widespread rhizomes, the identification of key regulatory genes in the bamboo shoot and rhizome growth control will provide important genetic resources for the genetic manipulation of plant height. In this study, we characterized 31 
GATA factors from moso bamboo. More importantly, the gene expression of PeGATAs is closely related to the development of rhizome tissues and the rapid-growth of bamboo shoots. Moreover, the gene expression of PeGATAs might be regulated by the phytohormone-GA and ABA in bamboo. In addition, functional characterization of PeGATA26 in Arabidopsis provides insight into how PeGATAs might regulate plant height from Arabidopsis to bamboo via downstream of the GA signaling pathway. In summary, our results provide evidence that the GATA transcription factor might regulate the development of rhizome tissues and the rapid-growth of bamboo shoots.

Supplementary Materials: Supplementary materials can be found at http://www.mdpi.com/1422-0067/21/1/14/s1.

Author Contributions: T.W., C.L., and L.M. conceived the ideas. T.W., Y.R. and W.W. performed the experiments. T.W., Y.Y., S.L. and Z.Z. contributed to data analysis. T.W. and L.M. wrote the manuscript. All authors have read and agreed to the published version of the manuscript.

Funding: This work was supported by the National Key R\&D Program of China (No. 2018YFD0600101 to Y.R.), the National Natural Science Foundation of China (Nos. 31500258 and 31741025 to L.M.), the Outstanding Youth Research Talents Development Program in Fujian Province University (to L.M.), and the Outstanding Youth Research Talents Program of FAFU (No. KXJQ17011 to L.M.), the Scientific Research Foundation of the Graduate School of FAFU (to T.W.). The funders had no role in the experimental design, data analysis, decision to publish, or preparation of the manuscript.

Acknowledgments: The authors thank Zhenguo Xu from Guangxi Forestry Research Institute for the certification of moso bamboo samples, and for producing and kindly providing space for storing the voucher specimen.

Conflicts of Interest: The authors declare no conflict of interest.

\section{References}

1. Wang, T.; Li, Q.; Lou, S.; Yang, Y.; Peng, L.; Lin, Z.; Hu, Q.; Ma, L. GSK3/shaggy-like kinase 1 ubiquitously regulates cell growth from Arabidopsis to Moso bamboo (Phyllostachys edulis). Plant Sci. 2019, 283, $290-300$. [CrossRef] [PubMed]

2. Wang, T.; Wang, H.; Cai, D.; Gao, Y.; Zhang, H.; Wang, Y.; Lin, C.; Ma, L.; Gu, L. Comprehensive profiling of rhizome-associated alternative splicing and alternative polyadenylation in moso bamboo (Phyllostachys edulis). Plant J. 2017, 91, 684-699. [CrossRef] [PubMed]

3. Lowry, J.A.; Atchley, W.R. Molecular evolution of the GATA family of transcription factors: Conservation within the DNA-binding domain. J. Mol. Evol. 2000, 50, 103-115. [CrossRef] [PubMed]

4. Scazzocchio, C. The fungal GATA factors. Curr. Opin. Microbiol. 2000, 3, 126-131. [CrossRef]

5. Katsumura, K.R.; Bresnick, E.H.; Group, G.F.M. The GATA factor revolution in hematology. Blood 2017, 129, 2092-2102. [CrossRef] [PubMed]

6. Patient, R.K.; McGhee, J.D. The GATA family (vertebrates and invertebrates). Curr. Opin. Genet. Dev. 2002, 12, 416-422. [CrossRef]

7. Crispino, J.D.; Horwitz, M.S. GATA factor mutations in hematologic disease. Blood 2017, 129, $2103-2110$. [CrossRef]

8. Teakle, G.R.; Gilmartin, P.M. Two forms of type IV zinc-finger motif and their kingdom-specific distribution between the flora, fauna and fungi. Trends Biochem. Sci. 1998, 23, 100-102. [CrossRef]

9. Reyes, J.C.; Muro-Pastor, M.I.; Florencio, F.J. The GATA family of transcription factors in Arabidopsis and rice. Plant Physiol. 2004, 134, 1718-1732. [CrossRef]

10. Zhang, C.; Hou, Y.; Hao, Q.; Chen, H.; Chen, L.; Yuan, S.; Shan, Z.; Zhang, X.; Yang, Z.; Qiu, D.; et al. Genome-wide survey of the soybean GATA transcription factor gene family and expression analysis under low nitrogen stress. PLoS ONE 2015, 10, e0125174. [CrossRef]

11. Zhang, Z.; Ren, C.; Zou, L.; Wang, Y.; Li, S.; Liang, Z. Characterization of the GATA gene family in Vitis vinifera: Genome-wide analysis, expression profiles, and involvement in light and phytohormone response. Genome 2018, 61, 713-723. [CrossRef] [PubMed]

12. Tremblay, M.; Sanchez-Ferras, O.; Bouchard, M. GATA transcription factors in development and disease. Development 2018, 145, dev164384. [CrossRef] [PubMed]

13. Terzaghi, W.B.; Cashmore, A.R. Light-regulated transcription. Annu. Rev. Plant Physiol. Plant Mol. Biol. 1995, 46, 445-474. [CrossRef] 
14. Daniel-Vedele, F.; Caboche, M. A tobacco cDNA clone encoding a GATA-1 zinc finger protein homologous to regulators of nitrogen metabolism in fungi. Mol. Gen. Genet. 1993, 240, 365-373. [PubMed]

15. Chen, H.; Shao, H.; Li, K.; Zhang, D.; Fan, S.; Li, Y.; Han, M. Genome-wide identification, evolution, and expression analysis of GATA transcription factors in apple (Malus×domestica Borkh.). Gene 2017, 627, 460-472. [CrossRef] [PubMed]

16. Hudson, D.; Guevara, D.R.; Hand, A.J.; Xu, Z.; Hao, L.; Chen, X.; Zhu, T.; Bi, Y.M.; Rothstein, S.J. Rice cytokinin GATA transcription Factor1 regulates chloroplast development and plant architecture. Plant Physiol. 2013, 162, 132-144. [CrossRef]

17. Richter, R.; Bastakis, E.; Schwechheimer, C. Cross-repressive interactions between SOC1 and the GATAs GNC and GNL/CGA1 in the control of greening, cold tolerance, and flowering time in Arabidopsis. Plant Physiol. 2013, 162, 1992-2004. [CrossRef]

18. Shikata, M.; Matsuda, Y.; Ando, K.; Nishii, A.; Takemura, M.; Yokota, A.; Kohchi, T. Characterization of Arabidopsis ZIM, a member of a novel plant-specific GATA factor gene family. J. Exp. Bot. 2004, 55, 631-639. [CrossRef]

19. Liu, P.-P.; Koizuka, N.; Martin, R.C.; Nonogaki, H. The BME3 (Blue Micropylar End 3) GATA zinc finger transcription factor is a positive regulator of Arabidopsis seed germination. Plant J. 2005, 44, 960-971. [CrossRef]

20. Fu, Y.H.; Marzluf, G.A. nit-2, the major nitrogen regulatory gene of Neurospora crassa, encodes a protein with a putative zinc finger DNA-binding domain. Mol. Cell Biol. 1990, 10, 1056-1065. [CrossRef]

21. Richter, R.; Behringer, C.; Müller, I.K.; Schwechheimer, C. The GATA-type transcription factors GNC and GNL/CGA1 repress gibberellin signaling downstream from DELLA proteins and PHYTOCHROME-INTERACTING FACTORS. Genes. Dev. 2010, 24, 2093-2104. [CrossRef] [PubMed]

22. Luo, X.-M.; Lin, W.H.; Zhu, S.; Zhu, J.Y.; Sun, Y.; Fan, X.Y.; Cheng, M.; Hao, Y.; Oh, E.; Tian, M.; et al. Integration of light-and brassinosteroid-signaling pathways by a GATA transcription factor in Arabidopsis. Dev. Cell 2010, 19, 872-883. [CrossRef] [PubMed]

23. Richter, R.; Behringer, C.; Zourelidou, M.; Schwechheimer, C. Convergence of auxin and gibberellin signaling on the regulation of the GATA transcription factors GNC and GNL in Arabidopsis thaliana. Proc. Natl. Acad. Sci. USA 2013, 110, 13192-13197. [CrossRef] [PubMed]

24. Buzby, J.S.; Yamada, T.; Tobin, E.M. A light-regulated DNA-binding activity interacts with a conserved region of a Lemna gibba rbcS promoter. Plant Cell 1990, 2, 805-814.

25. Carre, I.A.; Kay, S.A. Multiple DNA-Protein Complexes at a Circadian-Regulated Promoter Element. Plant Cell 1995, 7, 2039-2051. [CrossRef]

26. Naito, T.; Kiba, T.; Koizumi, N.; Yamashino, T.; Mizuno, T. Characterization of a unique GATA family gene that responds to both light and cytokinin in Arabidopsis thaliana. Biosci. Biotechnol. Biochem. 2007, 71, 1557-1560. [CrossRef]

27. Li, L.; Cheng, Z.; Ma, Y.; Bai, Q.; Li, X.; Cao, Z.; Wu, Z.; Gao, J. The association of hormone signalling genes, transcription and changes in shoot anatomy during moso bamboo growth. Plant Biotechnol. J. 2018, 16, 72-85. [CrossRef]

28. Zhang, H.; Wang, H.; Zhu, Q.; Gao, Y.; Wang, H.; Zhao, L.; Wang, Y.; Xi, F.; Wang, W.; Yang, Y.; et al. Transcriptome characterization of moso bamboo (Phyllostachys edulis) seedlings in response to exogenous gibberellin applications. BMC Plant Biol. 2018, 18, 125. [CrossRef]

29. Zhao, H.; Lou, Y.; Sun, H.; Li, L.; Wang, L.; Dong, L.; Gao, Z. Transcriptome and comparative gene expression analysis of Phyllostachys edulis in response to high light. BMC Plant Biol. 2016, 16, 34. [CrossRef]

30. Liu, H.-L.; Wu, M.; Li, F.; Gao, Y.-M.; Chen, F.; Xiang, Y. TCP Transcription Factors in Moso Bamboo (Phyllostachys edulis): Genome-Wide Identification and Expression Analysis. Front. Plant Sci. 2018, 9, 1263. [CrossRef]

31. Cheng, X.; Xiong, R.; Yan, H.; Gao, Y.; Liu, H.; Wu, M.; Xiang, Y. The trihelix family of transcription factors: Functional and evolutionary analysis in Moso bamboo (Phyllostachys edulis). BMC Plant Biol. 2019, 19, 154. [CrossRef] [PubMed]

32. Hou, D.; Cheng, Z.; Xie, L.; Li, X.; Li, J.; Mu, S.; Gao, J. The R2R3MYB Gene Family in Phyllostachys edulis: Genome-Wide Analysis and Identification of Stress or Development-Related R2R3MYBs. Front. Plant Sci. 2018, 9, 738. [CrossRef] [PubMed] 
33. Kobayashi, K.; Obayashi, T.; Masuda, T. Role of the G-box element in regulation of chlorophyll biosynthesis in Arabidopsis roots. Plant Signal Behav. 2012, 7, 922-926. [CrossRef] [PubMed]

34. Wang, W.; Gu, L.; Ye, S.; Zhang, H.; Cai, C.; Xiang, M.; Gao, Y.; Wang, Q.; Lin, C.; Zhu, Q. Genome-wide analysis and transcriptomic profiling of the auxin biosynthesis, transport and signaling family genes in moso bamboo (Phyllostachys heterocycla). BMC Genomics 2017, 18, 870. [CrossRef] [PubMed]

35. Song, X.; Peng, C.; Zhou, G.; Gu, H.; Li, Q.; Zhang, C. Dynamic allocation and transfer of non-structural carbohydrates, a possible mechanism for the explosive growth of Moso bamboo (Phyllostachys heterocycla). Sci. Rep. 2016, 6, 25908. [CrossRef] [PubMed]

36. Peng, Z.; Li, L.; Zhao, Q.; Feng, Q.; Gao, Z.; Lu, H.; Hu, T.; Yao, N.; Liu, K.; Li, Y.; et al. The draft genome of the fast-growing non-timber forest species moso bamboo (Phyllostachys heterocycla). Nat. Genet. 2013, 45, 456-461. [CrossRef] [PubMed]

37. Sun, H.; Wang, S.; Lou, Y.; Zhu, C.; Zhao, H.; Li, Y.; Li, X.; Gao, Z. Whole-Genome and Expression Analyses of Bamboo Aquaporin Genes Reveal Their Functions Involved in Maintaining Diurnal Water Balance in Bamboo Shoots. Cells 2018, 7, 195. [CrossRef]

38. Jeong, M.J.; Shih, M.C. Interaction of a GATA factor with cis-acting elements involved in light regulation of nuclear genes encoding chloroplast glyceraldehyde-3-phosphate dehydrogenase in Arabidopsis. Biochem. Biophys. Res. Commun. 2003, 300, 555-562. [CrossRef]

39. Jiang, K.; Yung, V.; Chiba, T.; Feldman, L.J. Longitudinal patterning in roots: A GATA2-auxin interaction underlies and maintains the root transition domain. Planta 2018, 247, 831-843. [CrossRef]

40. Kollmer, I.; Werner, T.; Schmulling, T. Ectopic expression of different cytokinin-regulated transcription factor genes of Arabidopsis thaliana alters plant growth and development. J. Plant Physiol. 2011, 168, 1320-1327. [CrossRef]

41. Ravindran, P.; Verma, V.; Stamm, P.; Kumar, P.P. A Novel RGL2-DOF6 Complex Contributes to Primary Seed Dormancy in Arabidopsis thaliana by Regulating a GATA Transcription Factor. Mol. Plant 2017, 10, 1307-1320. [CrossRef] [PubMed]

42. He, C.Y.; Cui, K.; Zhang, J.G.; Duan, A.G.; Zeng, Y.F. Next-generation sequencing-based mRNA and microRNA expression profiling analysis revealed pathways involved in the rapid growth of developing culms in Moso bamboo. BMC Plant Biol. 2013, 13, 119. [CrossRef] [PubMed]

43. Wang, Y.; Gao, Y.; Zhang, H.; Wang, H.; Liu, X.; Xu, X.; Zhang, Z.; Kohnen, M.V.; Hu, K.; Wang, H.; et al. Genome-Wide Profiling of Circular RNAs in the Rapidly Growing Shoots of Moso Bamboo (Phyllostachys edulis). Plant Cell Physiol. 2019, 60, 1354-1373. [CrossRef] [PubMed]

44. Finn, R.D.; Tate, J.; Mistry, J.; Coggill, P.C.; Sammut, S.J.; Hotz, H.-R.; Ceric, G.; Forslund, K.; Eddy, S.R.; Sonnhammer, E.L.L.; et al. The Pfam protein families database. Nucleic Acids Res. 2008, 36, D281-D288. [CrossRef] [PubMed]

45. Thompson, J.D.; Gibson, T.J.; Plewniak, F.; Jeanmougin, F.; Higgins, D.G. The CLUSTAL_X windows interface: Flexible strategies for multiple sequence alignment aided by quality analysis tools. Nucleic Acids Res. 1997, 25, 4876-4882. [CrossRef] [PubMed]

46. Kumar, S.; Stecher, G.; Tamura, K. MEGA7: Molecular Evolutionary Genetics Analysis Version 7.0 for Bigger Datasets. Mol. Biol. Evol. 2016, 33, 1870-1874. [CrossRef] [PubMed]

47. Marchler-Bauer, A.; Bo, Y.; Han, L.; He, J.; Lanczycki, C.J.; Lu, S.; Chitsaz, F.; Derbyshire, M.K.; Geer, R.C.; Gonzales, N.R.; et al. CDD/SPARCLE: Functional classification of proteins via subfamily domain architectures. Nucleic Acids Res. 2017, 45, D200-D203. [CrossRef]

48. Bailey, T.L.; Williams, N.; Misleh, C.; Li, W.W. MEME: Discovering and analyzing DNA and protein sequence motifs. Nucleic Acids Res. 2006, 34, W369-W373. [CrossRef]

49. Lescot, M.; Déhais, P.; Thijs, G.; Marchal, K.; Moreau, Y.; Van de Peer, Y.; Rouzé, P.; Rombauts, S. PlantCARE, a database of plant cis-acting regulatory elements and a portal to tools for in silico analysis of promoter sequences. Nucleic Acids Res. 2002, 30, 325-327. [CrossRef]

50. Zahur, M.; Maqbool, A.; Irfan, M.; Barozai, M.Y.; Qaiser, U.; Rashid, B.; Husnain, T.; Riazuddin, S. Functional analysis of cotton small heat shock protein promoter region in response to abiotic stresses in tobacco using Agrobacterium-mediated transient assay. Mol. Biol. Rep. 2009, 36, 1915-1921. [CrossRef] 
51. Trapnell, C.; Roberts, A.; Goff, L.; Pertea, G.; Kim, D.; Kelley, D.; Pimentel, H.; Salzberg, S.; Rinn, J.; Pachter, L. Differential gene and transcript expression analysis of RNA-seq experiments with TopHat and Cufflinks. Nat. Protoc. 2012, 7, 562-578. [CrossRef] [PubMed]

(c)

(C) 2019 by the authors. Licensee MDPI, Basel, Switzerland. This article is an open access article distributed under the terms and conditions of the Creative Commons Attribution (CC BY) license (http://creativecommons.org/licenses/by/4.0/). 